\title{
POSICIONAMIENTOS ÉTICOS EN EDUCACIÓN Y NUEVOS FORMATOS ESCOLARES PARA UNA PROTECCIÓN INTEGRAL DE LAS Y LOS ADOLESCENTES EN LA EDUCACIÓN SECUNDARIA OBLIGATORIA
}

\author{
Irma Leonor Kundt \\ (Universidad Nacional de la Patagonia San Juan Bosco)* \\ irmakundt@yahoo.com.ar
}

Recibido: 26/07/2012 Aceptado: 11/09/2012

\section{Resumen}

Este trabajo presenta un programa que crea en la acción nuevos formatos escolares. Comparte la construcción de un marco referencial en curso al interior de cuatro colegios públicos que integran una red, y la implementación de una experiencia piloto en uno de ellos. La misma se pone a prueba, en consideración y en diálogo para traccionar la transformación educativa en vista a los nuevos desafíos que impone pensar la educación secundaria y refundar la escuela abandonando los dispositivos pedagógicos de la modernidad. El agenciamiento de nuevos modos de hacer escuela y el estudio de la eficacia psíquica que cobran estas prácticas constituye su objetivo general.

El equipo asume profesionalmente la responsabilidad de la escuela en el riesgo que representa para los estudiantes la repitencia, constituyendo éste el problema que aborda y en el que centra su atención la intervención programada. Pone la atención en la enseñanza, fortalece y potencia la acción docente mediante la práctica entre varios y la generación de nuevos dispositivos de intervención, caso por caso, para respetar las trayectorias diferenciales y alojar la singularidad de los estudiantes desde el paradigma de la protección integral de sus derechos.

\section{Palabras claves}

Educación y premisas éticas - Derechos y protección integral - Nuevos formatos escolares.

* Especialista en Constructivismo y Educación- FLACSO-UAM. Diploma en Gestión de las Instituciones educativas. FLACSO. Profesora y Licenciada en Ciencias de la Educación. Universidad Nacional de la Patagonia San Juan Bosco. 


\section{Abstract}

This essay introduces a programme that designs new scholastic models in actions. It promotes discussion and the building of a referential framework inside four state schools which are part of a web, and the implementation of a singular experience in one of these schools. This experience will be tested and taken into consideration when promoting educational transformation. To think about secondary school and recover formal education become the new challenges, plus the necessity of giving up modern pedagogical outlooks. The focus is on designing new ways of building education and the study of their psychic effectiveness.

The staff takes responsibility for those students who are close to fail school, so this is the crucial matter of the programme. Teaching, teamwork, practices and new ways of solving problems become fundamental to deal with students' singularities and their achievements in formal education. All studied from a paradigm of rights integral protection.

\section{Key words}

Education - Ethic premises - Rights and integral protection - New scholastic models

\section{Introducción}

En el marco de las Leyes de Educación Nacional № 26.206 y Provincial VIII No 91 , que establecen la obligatoriedad de la educación secundaria, la política educativa del actual Ministerio de Educación de la Provincia del Chubut orienta las acciones pedagógicas de las escuelas proponiendo el trabajo en red.

La Circular № 1 explicita: "Entendemos por Red de escuelas, un agrupamiento que constituye un tipo de institucionalidad diferente; implica la disponibilidad de cada escuela para un trabajo cooperativo que aborde problemáticas comunes y en la que se comparten recursos, humanos, materiales y simbólicos". (Marco Organizativo Para La Escuela Secundaria Obligatoria Del Chubut; 2012. p. s. n)

En el mismo documento la política educativa recupera, entre otros, el concepto de inclusión instando a pensar y hacer "una escuela a la que todos los adolescentes puedan acceder y sean reconocidos. (...) Una escuela inclusiva es la que "hace lugar" a todos; no existe en el nuevo secundario espacio para escuelas expulsivas, que respondan a una lógica selectiva de los mejores". (2012: p. s. n)

Estas políticas educativas consideran y explicitan, en esta circular, que una educación de calidad "parte de ofertas variadas en la que las escuelas suman sus recursos y los combinan; compartiendo la tarea con otras escuelas para generar aprendizajes duraderos en todos sus estudiantes". (2012: p. s. n)

Los programas y proyectos que trabaja la red, encarando problemas comunes, responden a una convocatoria del Ministerio. Convocatoria a pensar 
en la acción nuevos formatos escolares, y a revisar las reglamentaciones vigentes para el Nivel, a los efectos de iniciar la transformación de la Educación Secundaria.

Este trabajo presenta uno de esos programas, que específicamente se inscribe, entre los lineamientos del actual Ministerio de la Provincia del Chubut (1), dentro del eje Nuevos formatos institucionales. El programa comparte, desde sus inicios, la idea del desencuentro de las escuelas con las nuevas subjetividades que la habitan (Sztulwark y Duschatzky, 2010) y reconoce las limitaciones de muchas propuestas que, aunque significativas, "continúan dentro de un formato escolar que no logra generar una innovación porque no modifica la dinámica escolar interna, ni respeta las trayectorias -no lineales- de los estudiantes". (Circular № 1. Ministerio de educación de la provincia del Chubut. Año 2012. p. s. n). Por lo mismo el trabajo de los equipos sí procura modificar la dinámica escolar, alterar formatos escolares y respetar las trayectorias reales de los chicos. Directivos y docentes lo hacen alojando la convocatoria y generando encuentros para la discusión, la que se anticipa a la acción, la acompaña y la revisa procurando los reajustes.

En un principio la discusión expone el desencuentro actual entre los principios rectores de las políticas y la existencia de prácticas de exclusión y segregación al interior de las escuelas públicas. La red se propone desnudarlas como tarea insoslayable para pensar modos legítimos de incluir. Los equipos acuerdan que es preciso pensar y crear estos modos dentro del paradigma de la protección integral de los derechos de niños, niñas y adolescentes, respetando las trayectorias de todos los alumnos y, en el programa diseñado, creando dispositivos de intervención para repitentes con sobre edad, tradicionalmente desatendidos y rechazados.

\section{Estado de situación de la provincia del Chubut en relación a la atención de estudiantes de la educación secundaria con repitencia reiterada en su trayectoria escolar}

En el marco de la Ley Federal de educación № 24.195, el equipo técnico de la provincia del Chubut, integrado, entre otros, por el actual Ministro de Educación Prof. Luis Zaffaroni, entonces Director General de Educación General Básica, y la actual Subsecretaria de Política, Gestión y Evaluación de la educación, Prof. Saida Paredes, elaboraron y pusieron en marcha el Proyecto "Itinerarios Educativos para adolescentes del Tercer Ciclo" (2).

Hoy es preciso referenciar esta experiencia para describir brevemente el estado de situación de la provincia en relación a la atención de estudiantes de la educación secundaria con repitencia reiterada en su trayectoria escolar, y evaluar si es legítimo darle continuidad a este proyecto, en el marco de la Ley 26.206 que establece la obligatoriedad de la educación secundaria y extiende su duración a seis años. Se hace necesario revisar si sus lineamientos políticos 
y las prácticas concretas como dispositivos de reingreso a las que dieron lugar, y están aún vigentes, resultan congruentes con la Ley de Protección integral de la niñez, la adolescencia y la familia, $N^{\circ} 4347$, de la Legislatura De La Provincia Del Chubut, ya vigente desde 1997.

Entre los fundamentos del proyecto que crea los Itinerarios, leemos:

...el fracaso escolar, en un contexto social adverso (...) significa un obstáculo medular, a la hora de replantear y fortalecer el rol social de la Escuela; la educación como herramienta de transformación social, debería propender antes que a "integrar lo que se expulsa" a "evitar la exclusión" (Zaffaroni, Paredes, Landaeta y Coiro. 2005: 2) (3).

Uno de sus objetivos generales explicita: "Ofrecer una propuesta educativa de inclusión, a término, que posibilite a los jóvenes con sobre-edad cumplimentar su escolaridad obligatoria, para continuar estudios superiores y/o insertarse en el sistema laboral" (Zaffaroni, Paredes, Landaeta y Coiro. 2005: 3)

Y en cuanto a la organización, el documento especifica:

Los itinerarios se desarrollarán en dos años (...) en horario vespertino, a partir de las 18.00 hs y hasta las 22.15 hs; (...) de ser necesaria la extensión horaria se tomarán las últimas horas, dadas las características de los adolescentes que concurrirán a los itinerarios y las condiciones edilicias, previendo que en la mayoría de los casos, se comparta el edificio escolar. (Zaffaroni, Paredes, Landaeta y Coiro. 2005: 10)

En el trabajo de discusión, iniciado primeramente por los equipos directivos de los colegios que integran esta red, y extensivo poco a poco al plantel estable de los mismos, con la incorporación de los Profesores en orientación y tutoría (POT), preceptores y profesores de enseñanza practica (PEP), se destaca que los itinerarios no resultaron ser inclusivos, en tanto dieron lugar al agrupamiento de estudiantes procedentes de diferentes instituciones de origen, y que por su sobre edad no fueron absorbidos por las mismas dentro de la población de repitentes matriculados, viéndose en la obligación de migrar hacia trayectos diferenciados que funcionaron, y funcionan aún hoy, muchas veces en centros alejados de su residencia familiar, lo que los indujo, e induce, a abandonar la escolaridad.

El agravante de estos itinerarios es que se destinaron a una matrícula de estudiantes pobres. Entre los antecedentes y diagnóstico del proyecto, así lo especifica el documento, se recuperan experiencias de los informes de las direcciones de escuelas en general, que presentan "rasgos comunes" orientadas a abordar las problemáticas inherentes a la matrícula en riesgo: 
(...) fundamentalmente se trabaja con una población marginal, alumnos provenientes de hogares carenciados con situaciones familiares conflictivas, inmersas en un contexto de violencia y discriminación que se acentúa con el fracaso escolar, operando como determinante irreversible, en cuanto a la posibilidad de mejorar la calidad de vida y las condiciones de trabajo (...) (Zaffaroni, Paredes, Landaeta y Coiro. 2005: 4)

Por otro lado la propuesta, surgida en el marco de la Educación General Básica obligatoria, cuya última cohorte ha sido en el ciclo lectivo 2009, desatiende hoy a los adolescentes mayores que se encuentran dentro de la educación secundaria orientada.

En 2012, la apertura de Itinerarios no ha sido presupuestada en la previsión de algunos colegios y por lo tanto los estudiantes propios con sobre edad deben migrar hacia instituciones que sí los tienen y que resultan insuficientes en relación a la demanda.

Para algunas escuelas los itinerarios implican el empleo de un mayor número de aulas, de las que no siempre disponen y por lo mismo suelen organizarlos en horario vespertino (tal como lo explicita el proyecto analizado, en párrafo ya citado), incrementando situaciones de riesgo para adolescentes entre 14 y 18 años a los que es preciso restituir el derecho a la educación desde el principio rector de la prioridad absoluta y la protección integral.

En tiempo de cambios no solo es preciso ver y atender a las numerosas escenas que desnudan el drama de la escuela en tiempos de destitución institucional y despojo de las certezas, es necesario re peguntar, desde las prácticas de cada día y la reflexión didáctica, desde las políticas educativas que hoy se proponen: ¿cuál es el rumbo?. Nosotros, los pedagogos... ¿hacia dónde vamos, en qué dirección? ¿Cuáles son las coordenadas éticas escogidas?

\section{Partir de nuevas premisas y en otra dirección}

Una primera premisa central para pensar desde la red, y que constituye un basamento de la experiencia piloto como propuesta radicalmente diferente a la de los Itinerarios, es que no puede haber inclusión verdadera en espacios segregados. Si alguna vez nos pareció que no hay derecho a los agrupamientos en grados a, b, c, d, reservados estos últimos a alumnos con dificultades en su trayectoria escolar, no parece claro por qué de repente no se nos presentó del mismo modo la creación de esos itinerarios.

Aunque el trabajo de la red atraviesa distintos momentos en cada una de las instituciones que la integran, lo que responde a tiempos y dinámicas institucionales diferentes, a sus culturas escolares, a las formas de trabajo de los equipos de gestión, al estado de la formación docente y a sus mayores o menores resistencias respecto a la necesidad de habitar y hacer escuela 
desde un nuevo paradigma, necesariamente distante del que dio origen a las instituciones modernas, una segunda premisa unifica los esfuerzos: Decidir un posicionamiento ético resulta indelegable e impostergable.

En 1990, con la ratificación de la Convención sobre los Derechos del Niño se reconoce en forma explícita que todos/as los niños, niñas y adolescentes son sujetos de derechos, y a su vez, el Estado argentino, la sociedad y las familias se comprometen a tratarlos como tales. En el marco de este compromiso se deben adecuar las medidas legales, administrativas, normativas y reglamentarias para dar efectividad a los derechos humanos. El compromiso obliga a garantizarles la protección integral y el ejercicio pleno de sus derechos sin ningún tipo de discriminación. El rechazo de toda forma de discriminación representa en la actualidad una obligación jurídica, un imperativo social y un desafío para la educación. (Correia y Díaz, 2008: 6)

Tradicionalmente la falta de acreditación de los espacios curriculares presenta con el desaprobado las dificultades del estudiante en su trayectoria escolar, responsabilizándolo por el fracaso (Valdez, 2001). El programa pone la atención en la enseñanza y el docente, corre así la responsabilidad y la mirada puesta en el alumno y los problemas de aprendizaje.

La recuperación de un primer caso, (Sebastián), expone las ideas iniciales que despertaron el interés y el pasaje de un estado de preocupación al ejercicio de pensar en la acción el programa diseñado. Este pasaje a la acción pone de manifiesto cómo la experiencia innovadora crea oportunidades de aprendizaje no sólo para los alumnos destinatarios del programa sino para los equipos de trabajo que lo sostienen y comparten al hacerlo, en palabras de Graciela Frigerio, una tercera premisa: "abstenerse de toda declaración de impotencia".

Esta autora la destaca como prohibición necesaria para los profesionales de la educación que hoy trabajan en situaciones de borde, y añade que es preciso asumir, al mismo tiempo, reglas éticas que califica como habilitadoras: "prioridad a la palabra, a la invención, a la reflexión que facilita una elaboración que obstaculice la repetición de lo mismo cuando lo mismo es fuente de sufrimiento". (Frigerio, 2003: 13)

En relación a ello, al momento de pensar los efectos de la repitencia, se presentó en la discusión de trabajo, la imagen reveladora de espectadores que miran una película desagradable por segunda vez. La miran sin opción de eludir la obligación de la repetición.

Se pensaron las reacciones que provocaría en un grupo de docentes pasar por esta experiencia en una presentación ficcional cuyo propósito fuera el registro de sus sensaciones y vivencias. Se acordó que sería un buen ejercicio para realizar en reunión de horas institucionales, provocaría probablemente una gran incomodidad, malestar, sensaciones de pérdida de tiempo..., sobre todo 
si el film escogido fuese aburrido, impuesto y desvinculado, por la imposibilidad de elegirlo, del interés por mirarlo.

Cualquier espectador, en situaciones normales, podría hacer zapping o apagar el televisor.

La metáfora se presenta con gran poder de ilustración y desnuda su violencia: los chicos no deben ni pueden hacer zapping: irse del aula, evadir la clase escuchando música, "apagar la clase" y dormir sobre el banco... Si lo hacen se los sanciona punitivamente con llamados al orden o el peligro de volver a repetir.

Estos efectos ven potenciada y magnificada su perversidad ya que la naturalización de las prácticas institucionalizadas de la repitencia las torna invisibles. La invisibilidad opera arbitrariamente cuando la anulación de los procesos secundarios de pensamiento y simbolización deja a los alumnos sin posibilidad de resistir, o las resistencias encuentran como único modo de expresión la apatía, el desinterés generalizado, las conductas disruptivas, el ausentismo, abandono, o incluso, en el peor de los casos, la instalación de síntomas y mecanismos diversos de contra inteligencia a modo de única forma de escapar y preservar lo que la escuela con su propuesta reiterada excluye y ataca: el placer de aprender y la movilidad de pensar.

Luzuriaga, Isabel (1976) en su libro "La inteligencia contra sí misma" (4) plantea el concepto de contrainteligencia como un mecanismo inconsciente y activo, ligado a la pulsión de muerte. El concepto permite comprender un factor que opera en el fracaso escolar y la actitud del niño que se muestra apático, tranquilo. Desde estos aportes el olvido, la apatía, la desconexión, el no percibir, comprender y no conceptuar o elaborar los datos de la realidad, son manifestaciones sintomáticas de un proceso activo cuya finalidad es la de mantener al niño quieto. La energía psíquica del niño estaría empleada contra la inteligencia misma: olvidando en lugar de recordar, aislando en lugar de conectar, etc.

La arbitrariedad de la repitencia escolar extiende la duración de un film reiterado durante cinco a seis horas por día, de lunes a viernes, durante nueve meses. Si la película es mala el malestar es mayor.

La repetición del año implica generalmente la reiteración de la misma propuesta de enseñanza, la presentación de los mismos instrumentos confeccionados para los trabajos prácticos y evaluaciones, la reiteración de explicaciones con las mismas estrategias, el mismo docente, etc. Al mismo tiempo la pérdida del grupo de pares que a veces opera cómo único sostén del interés por la escuela.

¿Cómo no provocaría todo ello un conflicto manifiesto en comportamientos remisos a la propuesta escolar? ¿Cómo se puede asimilar arbitrariedad semejante? Tal vez en el desinterés o la desadaptación como forma defensiva podría el alumno encontrar una salida.

Es necesario asumir que a la deserción del estudiante le antecede el abandono escolar y su desatención por la abdicación de los adultos. Una quinta 


\section{premisa invita a "romper la inercia de la reiteración y habilitar posibilidades que los alumnos merecen".}

\section{Relato de preocupaciones e intervenciones a partir de un primer caso.}

Sebastián (5) es un alumno que habiendo finalizado las instancias complementarias correspondientes al ciclo lectivo 2010 queda con tres espacios pendientes del segundo año, sumados a dos espacios previos de primero, lo que le impide promover al tercer año de la escuela secundaria básica. El régimen de evaluación y acreditación presente establece la promoción considerando hasta dos espacios pendientes. En la escuela primaria había repetido un año.

El colegio al que asiste no tendrá itinerario de adolescentes en el ciclo 2012 y por lo tanto no podrá absorberlo por su sobre edad. Constituye la única oferta de educación secundaria en el barrio y éste se encuentra geográficamente alejado de otros centros de la ciudad.

Sebastián tiene dificultades, como muchos chicos, para ser integrado en establecimientos de otros barrios alejados de la residencia familiar, no sólo por limitaciones económicas de su grupo, sino por las rivalidades entre los chicos que los habitan y con quienes se producen enfrentamientos y situaciones incluso de extrema violencia.

Si bien estas razones resultan para el equipo directivo y la POT (6) suficientes para conjeturar que si Seba no es alojado en este establecimiento posiblemente abandone la escolaridad, es al mismo tiempo una postura ética para quienes lo integran desafiar la profecía, y detenerse a pensar su caso y el de tantos otros chicos cuya singularidad requiere la atención del uno por uno, en lugar de la aplicación de normas generales que sin la excepción lo dejará excluido.

El reconocimiento de que Sebastián está en su derecho impone a la escuela en relación a su cumplimiento, numerosas obligaciones: en forma impostergable la atención del caso singular mediante el diseño de una forma de acompañamiento que sostenga la trayectoria escolar real de Sebastián, en vez de que continúen prevaleciendo las trayectorias teóricas que las escuelas, con su tradición meritocrática y credencialista, validan y presentan como legítimas.

Resulta pertinente destacar la conceptualización y la diferencia entre las trayectorias teóricas y las trayectorias reales:

Se definen las primeras como aquellos itinerarios de los sujetos dentro del sistema que siguen la progresión lineal predicha y pronosticada en tiempos pautados por una periodización lineal tipo o modelo. La trayectoria teórica implica ingresar a tiempo, permanecer, avanzar un grado por año y promocionar. Las trayectorias reales, en cambio, se refieren a itinerarios no encauzados de los estudiantes, quienes transitan su escolarización de modos heterogéneos, variables y "siguiendo otro cauce" o "no encauzados" (Cir- 
cular $N^{0}$ 1. Ministerio de educación de la provincia del Chubut. Año 2012. p. s. n). (7).

En relación a ello y a partir del análisis de las trayectorias, se observa que un mismo alumno repite consecutivamente un mismo año en más de una oportunidad. A veces en una segunda repitencia aprueba y desaprueba los mismos espacios curriculares que en el ciclo anterior. Por lo mismo lo único verdaderamente repetido es el fracaso. En otros casos algunos alumnos repiten el año sólo por tres espacios pendientes de acreditación, y se manifiestan indisciplinados o con gran desinterés en los espacios que sí habían acreditado con calificación siete el año anterior, no esforzándose en lo más mínimo durante la repitencia, ni siquiera para acreditarlos con calificación cuatro como establece la normativa legal vigente ¿Podría pensarse como una forma defensiva contra la injusticia?

En la revisión de marcos normativos de evaluación y criterios de promoción y acreditación (Disposición del Ministerio de educación $\mathrm{N}^{\circ} 11 / 08$ ) se pensaron y diseñaron, en oposición a la arbitrariedad manifiesta que se desprende de la norma y de las prácticas a las que da lugar, nuevos formatos, líneas de acción e intervenciones para el caso:

1. Exceptuar a Sebastián de la aplicación de la normativa actual-Disposición $11 / 08$ - y legitimar su promoción al tercer año.

2. Realizar el análisis de su trayectoria y reconocer y acreditar todos los espacios cursados y aprobados, independientemente del ciclo en el que se produjera la repitencia.

3. Diseñar dispositivos de atención con formatos que incluyan experiencias de aula acelerada, aula flexible y atención personalizada para los espacios pendientes de acreditación.

4. Propiciar espacios de encuentro para presentar a Sebastían el programa y contar con su anuencia.

5. Revisar compromisos entre el alumno y el equipo de sostén, durante toda la experiencia.

6. Acompañar la decisión, la organización y la preparación de exámenes previos (que nunca fueron acreditados) mediante la organización de carpetas y secuencia de los exámenes.

7. Optimizar el aprovechamiento de talleres de PMI (8) o POA (9) tutorando a Sebastián.

8. Interiorizar de la propuesta de intervención a los docentes y solicitar asignación de tareas de enseñanza y orientación para el acompañamiento al alumno haciendo uso de sus horas institucionales.

9. Evaluar tareas y realizar control de asistencia del estudiante por espacio curricular en aquellos que curse regularmente. 


\section{Resultados obtenidos y esperados en la replicación del Programa.}

Durante el ciclo 2011 Sebastián regularizó su situación aprobando los exámenes, con lo que legitimó su promoción en el año superior inmediato (tercero secundaria). En el ciclo 2012 inició su escolaridad dentro del programa para regularizar la promoción al cuarto año, y así mantenerse junto al grupo de adolescentes más próximos a su edad. Recientemente Sebastían ha promovido el cuarto año luego de las instancias de examen que siguieron al receso invernal. Con aula acelerada, acompañamiento personalizado y escuela de invierno ha regularizado, promoviendo y alcanzando a su cohorte reduciendo en un solo año la sobre edad que lo incomodaba y ponía en riesgo.

La propuesta es replicable. Se enmarca dentro de la obligación, primeramente del Estado, de la escuela pública y de los profesionales de la educación que trabajan en ella, de garantizar el derecho a la educación sin exclusión, lo que impone atender al problema de alumnos repitentes con sobre edad con una propuesta al interior de la propia escuela.

Es posible sostener este propósito y cumplirlo con líneas de acción y políticas educativas enmarcadas en el paradigma de la protección integral que se acompañen con la asignación del presupuesto y el incremento de más recursos humanos que los equipos de trabajo requieren.

Para crear nuevos formatos escolares las buenas intensiones no alcanzan, como no alcanzan los principios declarativos a favor de los niños, las niñas y los adolescentes. Es preciso un nuevo encuadre de trabajo para erradicar prácticas de segregación basadas en el agrupamiento de estos estudiantes, sobre los que recaen estereotipias que no favorecen positivamente sus procesos de subjetividad, ni favorecen los posicionamientos del profesor en relación a ellos, frente a los que ya tiene bajas expectativas.

Con este proyecto en curso se espera promover acciones respetuosas de las trayectorias escolares y el mejoramiento, enriquecido en la acción, del diseño de un plan de sostén y/o reingreso para estudiantes con riesgo de abandono por repitencia y sobre edad que atienda el caso por caso, y en cada escuela, a diferencia de pensar que algunas escuelas deben funcionar como pasillos de reingreso (itinerarios) mientras otras se desentienden de sus estudiantes en riesgo.

La red de estas escuelas escoge asumir el desafío de "imaginar nuevas trayectorias escolares que contemplen la realidad de aquellos alumnos cuya trayectoria escolar real es discontinua y a los cuales es necesario reingresar". (Circular No 1: Marco Organizativo Para La Escuela Secundaria Obligatoria Del Chubut; 2012: p. s. n).

Se encuentra en acción y a la espera de que el Ministerio de Educación de la provincia del Chubut responda con la asignación de los recursos solicitados y en congruencia con la convocatoria efectuada. El éxito del programa depende en gran parte de ello. 


\section{Documentación de un segundo caso.}

El texto expuesto a continuación corresponde al registro en actas de una trayectoria programada en el marco de nuevos formatos escolares para una protección integral de las y los adolescentes en la educación secundaria obligatoria. En el momento de realizar este registro Roberto asistía al Colegio 732, institución que integra la red de escuelas. Lamentablemente ha desertado. Ha cumplido 18 años y por lo tanto es mayor de edad en la legislación vigente y sus padres aceptan, o más bien no encuentran los modos de garantizar el derecho a la educación de Roberto, y desisten de enviarlo al colegio ante el abandono de la escolaridad.

Igualmente resulta atinado compartir la intervención para el caso, de manera que permita pensar y avanzar en formatos flexibles y legitimados como posibles y válidos, y así los equipos de trabajo se atrevan a su diseño y puesta en marcha en forma anticipatoria y no cuando la deserción es inminente.

Comodoro Rivadavia, 1 de marzo de 2012

Estudiante: Roberto

En el día de la fecha conversamos con el alumno Roberto que viene al establecimiento a hacer entrega de la netbook. Manifiesta que dejará la escolaridad secundaria para realizar talleres en la Escuela de Oficio 769. Aprenderá allí mecánica durante cuatro meses y si aprueba aprenderá electricidad.

Le explicamos que la escuela secundaria es obligatoria y no puede abandonarla, pero al mismo tiempo queremos acompañar su interés y no obstaculizarlo. Resulta imperioso hacerle lugar al mismo pues Roberto está decidido a dejar la educación formal.

El primer problema que se presenta es la superposición de horarios entre las dos instituciones, lo que le impediría hacer la escuela de oficios en forma paralela a la educación secundaria.

Los cursos que realizaría se encuentran organizados entre las 12.00 y las $15.15 \mathrm{hs}$.

Roberto adeuda siete espacios curriculares de tercer año secundaria y dos asignaturas previas de segundo año. La Educación secundaria básica se encuentra organizada en el turno mañana entre las 7.20 y las 12.30 hs. Esta situación le impide promover al cuarto año que funciona en el establecimiento en el turno tarde entre las 13.00 y las 18.00 hs.

Con cualquiera de los dos turnos tendrá superposición de horarios si alentamos su interés, no obstante decidimos acompañar su decisión y para ello resolvemos organizar un itinerario singular que le permita continuar su escolaridad y aprender oficios.

En esta nueva organización escolar, decidimos:

Reconocer como válidas las acreditaciones logradas en los espacios curriculares del tercer año durante el ciclo lectivo 2011, a saber: ciencias naturales y educación física. 
Facilitar que recurse del tercer año sólo los espacios curriculares no acreditados y que se encuentren organizados entre las 7.20 y las 11.10 hs; horario en el que se retirará del establecimiento para tomar colectivo y llegar a tiempo a la otra institución.

(Su preceptora toma conocimiento de este dispositivo, lo entiende, comparte, interviene pensando y sugiriendo en el trabajo en equipo. Organizará horarios atendiendo a esta necesidad todo lo que sea posible. Ayudará a Roberto a organizar su agenda de obligaciones en relación a ellos trabajando en equipo junto a la POT).

Tomar asistencia en los espacios curriculares que integren su trayecto singular y dejar sin efecto la normativa que establece la asistencia de un $85 \%$ (Disposición Ministerial 11/08) a la escuela como un requisito de acreditación.

Mantener con Roberto contacto informal y formalizado en espacios de entrevistas que permitan sostener su interés, escuchar sus inquietudes, evaluar la experiencia y realizar los reajustes que sean necesarios.

Potenciar bajo la coordinación de la POT el aprovechamiento de los recursos humanos disponibles entre los MEP, los profesores asignados en talleres del PMI y los espacios de POA que se organicen con horas institucionales para que Roberto pueda aprender y acreditar las asignaturas previas, cursar a contra turno lo que no sea posible por la mañana y de este modo no se retrase en la escolaridad alejándose en edad de su cohorte y grupo de pares.

El equipo directivo iniciará y sostendrá el contacto con la escuela de oficios, atento inicialmente a establecer un sistema de equivalencias interno, y a efectos de esta intervención, entre los talleres de oficio y los espacios curriculares afines como Tecnología, favoreciendo la acreditación de los procesos de aprendizaje que el estudiante realice fuera de la escolaridad formal.

Informar a los padres del dispositivo de acompañamiento y realizar un acta acuerdo en la que queden definidos los horarios de cursada, ingreso y salida del estudiante.

Roberto se manifiesta muy conforme con la solución propuesta y se muestra animado y comprometido a realizar este recorrido.

\section{Navegar sobre un nuevo barco y en otra dirección}

Suele sentirse la añoranza y el reclamo constante por la reposición de un orden perdido, instituido. Y al mismo tiempo es posible ver que ese orden es excluyente. Los chicos, con sus formas de rebeldía, parecen denunciarlo.

¿Quieren crecer en desorden, vivir sin pautas? ¿O aceptarían otras pautas y un orden no excluyente?

Tal vez para ellos el problema se presenta, y nos lo presentan, porque se resisten a soportar las viejas condiciones. Si resistir encuentra una salida en la renuncia, el abandono, la deserción, el desafío y el deber para los docentes es cómo lograr que no desistan, lograrlo sin doblegarlos. 
Lo justo es pensar nuevas condiciones, que impliquen muchos modos, y entre otras cosas, el uso y la apropiación de la escuela como espacio público para las nuevas formas de ser y estar que tienen los chicos.

Es posible agenciar una intervención educativa saliendo de lo propiamente curricularista y disciplinario. Éticamente, resulta oportuno reconocer que hacerlo nos cambia el norte de lo "propiamente" educativo que ya no puede ser únicamente la enseñanza de las disciplinas. Es preciso subir a otro barco ante la emergencia de situaciones y escenas que desnudan el drama de la vieja nave. Sólo agenciando nuevas intervenciones no sentiremos la impotencia del náufrago.

El tercer caso escogido presenta una crónica a modo de un final abierto para no clausurar con conclusiones la invitación a seguir pensando.

Federico dejó la escuela. Vuelve ocasionalmente. Luego de varios meses se acerca a la dirección.

Directora: - ¡iQué bueno verte Federico!!

Al saludo responde inmediatamente con un pedido: -Yo quería saber si puedo participar de la muestra. Si puedo venir al gimnasio a practicar. Directora: - Si, que bueno, iiclaro que podés!! ¿qué necesitás?

Federico: - Usar las colchonetas.

Directora (en conversación con las porteras): -"Federico va a participar de la muestra, necesitamos saber en qué días y horarios está libre el gimnasio. Hay que abrirle y sacar las colchonetas del depósito. Les aviso para que no tenga problemas, si yo no estoy lo dejan entrar." ( $\mathrm{Y}$ dirigiéndose a Federico): - "si venís y no te dejan entrar, en otros turnos con otros porteros, le decís que hablaste conmigo. Que estás autorizado. Si no te dejan me avisas"

Noviembre de 2010:

Federico participó de la muestra. Lo ovacionaron. Es un excelente gimnasta.Se lo ve integrado al grupo.

Miriam, la profesora de educación física, también le hizo un lugar.

16 de Abril de 2011:

Es sábado, hay reunión de docentes en horas institucionales. Ingresa Federico a la cocina. Viene a buscar agua para Daniel, el profesor de educación física.

La directora le pregunta qué está haciendo en la escuela. Responde:"estoy ayudando al profesor con los chicos en las colchonetas"

La pregunta se dirige a indagar en las motivaciones que lo hacen venir a la escuela aunque abandone las clases, las ha abandonado por segunda vez, pues inició este año la modalidad semi presencial en horario vespertino, y lleva dos semanas ausente. 
Le cuenta a Daniel que ya no viene. Daniel le insiste que hable con los directivos y se reincorpore.

Con Daniel buscan que Federico tenga un lugar: ayudante alumno. Encontrarán el modo de agenciar una intervención que lo atienda saliendo de la oferta escolar igualitaria que lo deja desatendido. Lo buscarán fuera del dispositivo "clase". Es preciso tejer el soporte entre varios... Habrá que hacer equipo.

\section{Notas bibliográficas}

(1) Esos lineamentos a los que el trabajo refiere han sido comunicados a las escuelas mediante tres documentos recientes del Ministerio de educación de la Provincia del Chubut: Circular № 1, Marco Organizativo para La Escuela Secundaria Obligatoria Del Chubut; Carta a los Profesores, publicada en www.chubut.edu.ar en el mes de febrero de 2012, y Circular № 2, Para la acción institucional en red.

(2) El Itinerario Educativo para adolescentes del Tercer Ciclo inició su primera cohorte en algunas escuelas de las diferentes zonas de La Provincia del Chubut en marzo de 2005.

(3) El destacado con comillas en las dos expresiones dentro de la cita es del Documento del Equipo técnico de la Provincia del Chubut. Dirección General de EGB3 y Polimodal. Proyecto "Itinerarios Educativos para adolescentes del Tercer Ciclo". Zaffaroni, Paredes, Landaeta, y Coiro. 2005.

(4) Utilizando los descubrimientos del Psicoanálisis en este campo, la autora postula en esta obra, que tras una aparente inhibición intelectual que se da en niños físicamente sanos, operan por el contrario, procesos inconscientes muy activos e intelectuales, cuya finalidad es mantener al niño aislado, tanto de la comprensión de la realidad objetiva, como de la de su propio mundo interior, y ello debido a que, a veces la percepción inteligente de ambos mundos le causan conflictos y con ellos, ansiedad y sufrimiento psíquico.

(5) El nombre Sebastián documenta un primer caso y al mismo tiempo extiende y presenta en el relato la situación de diferentes alumnos y alumnas adolescentes que comparten la condición de tener sobre edad en relación al curso al que asisten por repitencia.

(6) La sigla POT nomina al Profesor en orientación y tutoría a partir del Decreto 146 que pone en vigencia y reglamenta la Ley de Educación Secundaria 26.206 en la provincia de Chubut.

(7) El destacado con comillas es del documento.

(8) La sigla PMI nomina al Programa Nacional de Mejoramiento Institucional que destina fondos a las escuelas públicas para la concreción de sus propios proyectos, inscriptos en su propuesta pedagógica y de gestión institucional. Se destinan parte de las horas cátedras a la atención de alumnos en los espacios curriculares de mayor riesgo.

(9) La sigla POA nomina al período durante el año destinado a la orientación y acompañamiento de los estudiantes en la preparación de exámenes pendientes. Los docentes generan oportunidad y formas alternativas de acreditación con horas cátedras destinadas a tal fin. 


\section{Referencias bibliográficas}

- $\quad$ Correia, M y Llaiquel, R. (2002). Ley de Protección Integral de la Niñez, la Adolescencia y la Familia. Principios Rectores. Niños, Menores e Infancias, № 1 año 1 . Universidad Nacional de La Plata. Argentina. Publicación del Instituto de Derechos del Niño. Facultad de Ciencias jurídicas y Sociales.

- $\quad$ Correia, M y Diaz, S. (2008). ¿Qué Significa Pensar los Niños, las Niñas y Adolescentes como Sujetos de Derechos en la Formación Docente? En BONINI, Elsa (Coord.) Problemáticas y Perspectivas de la Formación Docente. Páginas 4/11. Primera Edición CD ROM, Abril de 2008. Comodoro Rivadavia. Chubut. Editor: Universidad Nacional de la Patagonia San Juan Bosco. ISBN 978-950-763-083-5

- Constitución Nacional de la República Argentina Convención Nacional Constituyente, ciudad de Santa Fe, 22 de agosto de 1994.

- Duschatzky, S. y Corea, C. (2002). Chicos en banda. Los caminos de la subjetividad en el declive de las instituciones. (1ra edición). Buenos Aires: Editorial Paidós.

- Frigerio, G. (2006). Hacer del borde el comienzo de un espacio. Infancias y adolescencias. Teorías y experiencias en el borde. La educación discute la noción de destino, 50 , 6-14. Buenos Aires: Ediciones Novedades Educativa. Colección ensayos y experiencias.

- Kundt., I. y Gago, A. (2012). El agenciamiento de una intervención educativa para que un sujeto no habite la situación de exclusión social. En KUNDT, I. Habitar y hacer escuela. Buenos Aires: Vela al viento ediciones patagónicas.

- Luzuriaga, I. (1976). La inteligencia contra sí misma. El niño que no aprende. (3ra. Edición) España: Editorial Psique.

- Sztulwark, D y Duschatzky, S. (2010). Imágenes sobre lo no escolar. Diploma Superior en gestión educativa. Buenos Aires: FLACSO Argentina.

- Valdez, D (2001). Problemas de aprendizaje /problemas de enseñanza: hacia una recontextualización del problema. Año 13, № 128. Buenos Aires: Ediciones Novedades educativas. Colección ensayos y experiencias.

\section{Documentos oficiales:}

- Legislatura de la Provincia del Chubut. Ley 4347, De Protección Integral de la Niñez, la Adolescencia y la Familia. Rawson, Chubut-Argentina (1997).

- Ley de Educación de la Provincia del Chubut VIII- 91/10. Rawson, Chubut, Argentina. 4 de noviembre de 2010.

- Ministerio de Educación de la provincia de Chubut 2008. Disposición del N 11/08. Régimen de evaluación, promoción y acreditación para la educación secundaria y polimodal.

- Ministerio de Educación de la provincia de Chubut. 2012. Circular No 1: Marco Organizativo Para La Escuela Secundaria Obligatoria Del Chubut.

- Ministerio de Educación de la provincia de Chubut 2012. Circular N² 2: Para la acción institucional en red.

- Senado y Cámara de Diputados de la Nación Argentina. Ley de Educación Nacional № 26. 206. Sancionada el 14 de Diciembre de 2006 y Promulgada el 27 de Diciembre de 2006.

\section{Fuentes electrónicas}

- http://www.libreriapaidos.com/9788470305581/INTELIGENCIA+CONTRA+SI+MISMA \%2c+LA/. (Consultado: 22 /07/2012). 